\title{
Wissen und Begründen: Evidenz als umkämpfte Ressource in der Wissensgesellschaft
}

\author{
Einleitung
}

Sarah Ehlers und Karin Zachmann

Sich auf Evidenz im Sinne eines glaubwürdigen, verlässlichen und sozial konsentierten Wissens zu berufen, ist schwieriger geworden, seit sogenannte alternative Fakten und Fake News zirkulieren und allenthalben von einem postfaktischen Zeitalter die Rede ist. Die Entwertung von Fakten aber signalisiert eine potentiell gefährliche Abwertung der Wissenschaft, die überprüfbaren Fakten ihren Aufstieg verdankt. ${ }^{1}$ Nachdem moderne Wissensgesellschaften die Organisation von Wissen zur Grundlage des Zusammenlebens gemacht haben, verweist die Abwertung von Fakten auf eine weitreichendere gesellschaftliche Glaubwürdigkeitskrise.

Inwieweit ist das Ringen um die Gültigkeit von Wissen jedoch ein Phänomen der sogenannten Post-Truth-Ära? Was bedeutet es für die Wissensgesellschaft, wenn Wissen nicht mehr verlässlich ist und Evidenz nicht mehr überzeugen kann? Wie reagieren Wissenschaftler_innen und Expert_innen darauf, wenn das von ihnen produzierte Wissen in Frage gestellt wird? Wie dringen neue Akteur_innen in ein etabliertes institutionelles Feld ein und versuchen, ihrer Expertise Gültigkeit zu verschaffen? Aushandlungen um die Gültigkeit von Wissen, so die Leitthese des vorliegenden Bandes, sind ein im Laufe des 20. Jahrhunderts politisch brisanter werdendes Phänomen, das sich in multiplen Bereichen der Wissenschaft, der Technik, der Politik, der Medizin und der Gesellschaft zeigt: Wissen wird in modernen Gesellschaften nicht per se akzeptiert, sondern Wissensakteur_innen müssen überzeugen, um ihren Befunden Evidenz zu verleihen. Diese von uns als Evidenzpraktiken verstandenen Dynamiken zu untersuchen, ist die Aufgabe der hier versammelten Beiträge. In interdisziplinärer Perspektive widmen sich die Autor_innen dazu Beispielen aus der Medizin-, Kommunikations-, Wirtschafts-, Wissenschafts- und Umweltwelt-

1 Jill Lepore, After the Fact. In the History of Truth, a New Chapter Begins, in: The New Yorker, 14.3.2016: https:/www.newyorker.com/magazine/2016/03/21/the-inte rnet-of-us-and-the-end-of-facts [Stand: 24.4.2019]. 
forschung und kombinieren dabei die Analyse der jüngsten Geschichte mit aktuellen Phänomenen.

Die Wissenschaft begann ihren Siegeszug, indem sie mit dem Bezug auf überprüfbare Fakten ein neues Legitimationssystem schuf und damit neben die Autorität der Kirche trat. Als der Philosoph Immanuel Kant vor über 200 Jahren die Frage aufwarf: „Was kann ich wissen?“", wussten die Wissenschaftler seiner Zeit darauf eine klare Antwort: Fakten entdecken, sammeln, gewichten und interpretieren, das galt als Königsweg zu sicherem Wissen und wies wissenschaftlicher Evidenz die Macht zur Erklärung der Welt sowie das Potential zur Auflösung von Konflikten zu. Dabei konnte die Wissenschaft vom Autoritätsverlust der Religion profitieren und es wurde von ihr erwartet, deren Heilsversprechen durch ihren Wahrheitsanspruch ersetzen. Mit der Professionalisierung der Wissenschaft entstand im 19. Jahrhundert ein sozial homogener sowie durch Normen ${ }^{2}$ stabilisierter und überschaubarer Raum, in dem Fragen zugelassen und Glaubwürdigkeit verhandelt werden konnte.

Im Verlaufe des 20. Jahrhunderts veränderten sich diese Bedingungen der Wissensproduktion jedoch signifikant und insbesondere die gesellschaftliche Forderung nach Evidenz wurde zentral. Die besonders im letzten Viertel des vergangenen Jahrhunderts anschwellenden Diskurse um Risiko, Unsicherheit und Nichtwissen verweisen darauf, dass die Wissenschaften ihr Gewissheitsversprechen relativieren mussten und selbst zunehmend unter Legitimationszwänge geraten sind. ${ }^{3}$ Dabei trieb gerade die beunruhigende Erfahrung wachsender Unsicherheit das Verlangen nach eindeutiger Evidenz hervor. ${ }^{4}$ Gleichzeitig verwandelten sich, indem Wissen zu einer zentralen gesellschaftlichen Ressource geworden war, wissenschaftliche Unsicherheiten nun unmittelbar auch zu gesellschaftlichen Unsicherheiten und Risiken. ${ }^{5}$

Das Bedürfnis nach Evidenz hat also keineswegs ab-, sondern vielmehr zugenommen. Sichtbar wird das daran, dass seit ca. 30 Jahren Evidenzbasierung ein Schlagwort für neue Programme und Projekte geworden ist,

2 Gemeint sind hier Robert Mertons CUDOs-Normen.

3 Stefan Böschen u. Peter Wehling, Neue Wissensarten. Risiko und Nichtwissen, in: Sabine Maasen et al. (Hg.), Handbuch Wissenschaftssoziologie, Wiesbaden 2012, S. 317-327.

4 Stefan Böschen, Zur Einleitung. Fragile Evidenz - Wissenspolitischer Sprengstoff, in: Technikfolgenabschätzung - Theorie und Praxis 22, H. 3, November 2013, S. 49.

5 Ingo Schulz-Schaeffer u. Stefan Böschen, Einleitung, in: Stefan Böschen et al. (Hg.), Wissenschaft in der Wissensgesellschaft, Wiesbaden 2003, S. 9-23. 
die darauf abzielen, vorhandenes Wissen konsequenter als Grundlage praktischer Entscheidungen und Handlungen zu mobilisieren. In der Medizin hat die Karriere des Evidenzkonzepts schon vor fast 50 Jahren Fahrt aufgenommen und zur Entwicklung von Strategien geführt, um vorhandene Ergebnisse klinischer Studien als externe Evidenz in die Behandlungspraxis zu integrieren. ${ }^{6}$ Die Pädagogik und neuerdings auch das Management sind weitere Bereiche, in denen Evidenzbasierung als grundlegendes Qualitätskriterium aufgewertet worden ist. In der Politik kam die explizite Forderung nach Evidenzbasierung um die Jahrtausendwende auf mit dem Ziel, die schon lange etablierte Praxis problemorientierter, wissenschaftlicher Politikberatung zu verstetigen, zu systematisieren und auf Politikfolgenabschätzung und Politikevaluierung zu fokussieren. Evidenz verbindet sich hier mit der Erwartung, Politik zu versachlichen, damit die Qualität der Gesetzgebung zu verbessern und die Kosten der Regulierung zu verringern. ${ }^{7}$

Nicht nur für gesellschaftliche Teilbereiche, sondern auch für die Organisation des gesellschaftlichen Zusammenlebens generell erlangte Evidenz im Verlaufe des 20. Jahrhunderts eine zentrale Bedeutung. Das wiederum hat eine weit zurückliegende, dann aber lange unterbrochene Tradition. In der griechischen Polis transformierte Sokrates die Frage, wie Übereinstimmung zu gemeinschaftlich ausgehandelten Kompromissen auf der Agora zu erreichen sei, in die Aufgabe, Zustimmung zu vorgegebenen Lösungen durch Anerkennung des Wissens von Experten zu erlangen. Damit beging er, so kritisiert der Philosoph Bruno Latour, einen Kategorienfehler: „Auf den Bereich der Politik wendet er einen ,Wahrheitskontext ${ }^{\star}$ an, der in einen anderen Bereich gehört." ${ }^{8}$ Allerdings lag im Bezug auf Expertenwissen auch ein produktiver Aspekt, wenn es nicht, wie von Sokrates, missbraucht wurde, um Politik zu blockieren. Die Griechen etablierten mit dem geometrischen Beweis auch die ideale Vorstellung vom offenen $\mathrm{Zu}$ gang zu Wissen. ${ }^{9}$ Das unterstellte jedoch, dass die geometrischen Verhält-

6 Heiner Raspe, Eine kurze Geschichte der Evidenz-basierten Medizin in Deutschland, in: Medizinhistorisches Journal 53, H. 1, 2018, S. 71-82.

7 Sabine Weiland, Evidenzbasierte Politik zwischen Eindeutigkeit und Reflexivität, in: Technikfolgenabschätzung - Theorie und Praxis 22, H. 3, November 2013, S. 915.

8 Bruno Latour, Die Hoffnung der Pandora, Frankfurt am Main 2000, S. 303.

9 Darauf verweist Theodore Porter, Trust in Numbers. The Pursuit of Objectivity in Science and Public Life, Princeton 1996, S. 78, unter Bezug auf Amos Funkenstein, Theology and the Scientific Imagination from the Middle Ages to the Seventeenth Century, Princeton 1986, S. 358. 
nisse plausibel und überzeugend seien und Verständigung ermöglichten. Mithin lag in der gleichzeitigen Erfindung von Geometrie und Demokratie ein ambivalentes historisches Vermächtnis. Es materialisierte sich einerseits darin, dass die Autorität von Experten eine deliberative Politik oft unterband. Aber andererseits zeitigte es langfristige Wirkungen dergestalt, dass in modernen Massendemokratien gesichertes, im Sinne von anerkanntem Wissen die Basis von Konsensfindung darstellt. Nach dem Wissenschaftssoziologen Peter Weingart ist „Evidenzbegründung [...] nach wie vor Prinzip der Politik sowie der Beilegung oder Vermeidung von Konflikten, die sich aus widersprüchlichen Meinungen ergeben". ${ }^{10}$

Voraussetzung aber für diesen Modus der Konfliktbewältigung ist, dass es eine gemeinsame Basis für die Transformation von Wissen in Gewissheit gibt. Demokratische Gesellschaften brauchen nicht nur im Bereich der Wirtschaft eine gemeinsame Währung, um Dinge/Waren auszutauschen. Sie benötigen auch im Bereich der Politik und der Gesellschaft ein solches Medium, um Argumente auszutauschen und Auseinandersetzungen friedlich zu führen, sodass Einigungen herbeigeführt werden können. Diese gemeinsame Währung aber ist, so der Philosoph Michael Lynch, „reason as capacity to explain and to justify" ${ }^{11}$ Vernunft gestattet es, sich auf Fakten und Überzeugungen zu einigen, weil sie auf epistemischen Prinzipien beruht, die substantiell für wissenschaftliche Praktiken sind: Intersubjektivität, Transparenz, Wiederholbarkeit. Auch wenn epistemische Prinzipien letztlich nicht begründbar sind, ohne in den Zirkelschluss einer sich selbst beweisenden Vernunft zu verfallen, haben wir praktische Gründe, an die Verlässlichkeit dieser Prinzipien zu glauben. Sie eröffnen den Zugang zu Begründungen, die von einem gemeinsamen Standpunkt aus zugänglich sind. Damit ist ihre Anwendung grundlegend für das Funktionieren einer Demokratie. ${ }^{12}$

Aber die Berufung auf wissenschaftliche Praktiken als verlässliche Quellen unserer Evidenz ist keineswegs unumstritten. Die Diagnose vom postfaktischen Zeitalter markiert dabei eine Entwicklung, in der die Herausforderungen an Evidenz als gemeinsame Basis für Begründungen von Wissen und Entscheidungen stetig zunehmen. Die Gründe dafür sind vielfältig, die damit verbundenen Gefahren sind es auch.

10 Peter Weingart, „Wahres Wissen“ und demokratisch verfasste Gesellschaften, in: Aus Politik und Zeitgeschichte (APuZ) 67, H. 13, 2017, S. 11-16, hier S. 16.

11 Michael P. Lynch, In Praise of Reason, Cambridge u. London 2012, S. 31.

12 Ebd., Kapitel 5. 
Eine erste Gruppe von Gründen lässt sich im Bereich der Wissenschaften selbst ausmachen. Seit der zweiten Hälfte des 19. Jahrhunderts begannen sich markante Veränderungen in der Wissensproduktion abzuzeichnen, die der Philosoph Gregor Schiemann in seiner Arbeit über den Physiker Hermann von Helmholtz als „Wahrheitsgewissheitsverlust" kenntlich gemacht hat. ${ }^{13}$ Schiemann argumentiert, dass mit dem Übergang von der klassischen zur modernen Wissenschaft Erfahrungsdaten allmählich ihren Status als allgemeingültige Überprüfungsinstanz der Erkenntnis verloren und stattdessen Hypothesen zum festen Bestandteil wissenschaftlicher Erkenntnis aufrückten. Mit der Aufwertung von Hypothesen aber kam die Vorläufigkeit von Wissen ins Bewusstsein. Das wiederum unterminierte dessen Verlässlichkeit und gleichzeitig auch die Bedeutung von Fakten und empirischen Tatsachen als Quellen von Evidenz. Im 20. Jahrhundert nahm dieser Gewissheitsverlust weiter zu, da sich der hypothetische Charakter von Wissen durch die wachsenden Ansprüche an dessen prognostisches Potential weiter ausgeprägt hat. ${ }^{14}$ Diese Entwicklung hat sich auch dadurch verstärkt, dass die Wissenschaft sich als Mitverursacherin von Risiken in Gestalt nicht intendierter Nebenfolgen ihrer Ergebnisse zunehmend mit der Abschätzung zukünftig auftretender Auswirkungen befassen muss, so z.B. in der Gentechnik, bei neuen Therapien oder in der Energietechnik. ${ }^{15}$ Aussagen über die Zukunft aber sind inhärent unsicher, weil wir nicht wissen können, was in der Zukunft geschieht. ${ }^{16}$ Hier stoßen wir auf die unumgehbare Kontingenz.

Ganz neue Herausforderungen an Evidenz ergeben sich zweitens aus der Digitalisierung und der Entwicklung des Internets, das sich als zunehmend omnipräsentes Medium der Generierung und Vermittlung von Informationen als Grundlage von Wissen etabliert. Das schafft einerseits neue Zugangs- und Partizipationsmöglichkeiten. Andererseits erschwert es die Abgrenzung von Informationen und Wissen und verändert die Verfahren, in denen aus Informationen Wissen entsteht oder auch nur als solches

13 Gregor Schiemann, Wahrheitsgewissheitsverlust. Hermann von Helmholtz' Mechanismus im Anbruch der Moderne. Eine Studie zum Übergang von klassischer zu moderner Naturphilosophie, Darmstadt 1997, S. 138-140.

14 Eine soziologische Analyse der Verwissenschaftlichung der Zukunft bietet Mario Kaiser, Neue Zukünfte - Gegenwarten in Verzug, in: Sabine Maasen et al. (Hg.), Handbuch Wissenschaftssoziologie, Wiesbaden 2012, S. 395-408.

15 Wilde, Jessica, Ulrich Beck: Die Risikogesellschaft als Wegbereiter der Wissensgesellschaft? In: Anina Engelhardt u. Laura Kajetzke (Hg.), Handbuch Wissensgesellschaft. Theorien, Themen und Probleme, Bielefeld 2010, S. 35-42.

16 Nicholas Rescher, Ignorance. On the Wider Implications of Deficient Knowledge, Pittsburgh 2009. 
behauptet wird. ${ }^{17}$ Folglich wurde nicht nur der Zugang zu Wissen pluralisiert, sondern auch die Wissensproduzenten und damit das Angebot konkurrierender Wissensbestände. Grundlegend dafür ist die Ersetzung von Fakten durch Daten, die nicht mehr gesammelt, bearbeitet und begründet, sondern hoch- oder heruntergeladen werden. Im Ergebnis entsteht ein neuer Modus des Wissens, den Lynch als "Google-knowing“ von anderen Arten zu wissen unterscheidet, insbesondere vom Verstehen als einer individuellen, kognitiven Errungenschaft. ${ }^{18}$ Verstehen ist die Bedingung, um etwas zu erklären und zu begründen. Auf dieser Basis entsteht Evidenz als sozial konsentiertes Wissen und Grundlage für Entscheidungen. Mit "Google-knowledge" aber verlassen wir uns zunehmend auf Algorithmen, die „Wissen“ maschinell über das Korrelieren von Daten und Informationen generieren. Damit gibt es zwar einen Zugewinn an frei verfügbarem Wissen, doch die Frage der Relevanz und die Suche nach sinnhaften Erklärungen rückt in den Hintergrund.

Mit der Einbeziehung digitaler Techniken kommen zudem neue Akteur_innen und Aktanten ins Spiel, die die sozioepistemischen Arrangements der Evidenzierung weiter ausdifferenzieren. Das sind einerseits die Hardware- und Software-Entwickler_innen und die von ihnen bereitgestellten Programme, die neue Möglichkeiten der Erschließung und Verarbeitung von Informationen gestalten. Das sind andererseits die Nutzer_innen der digitalen Techniken, die z.B. als gemeinnützige oder kommerzielle Plattformbetreiber_innen, aber auch als engagierte Bürger_innen, Patient_innen, Konsument_innen etc. Informationen bereitstellen, spenden, sammeln, verkaufen und dabei beanspruchen, eine aus ihrer Perspektive angemessenere Evidenz zu generieren.

Auch die zunehmende Anzahl privatwirtschaftlich aktiver Anbieter kommodifizierter Evidenz trägt zur Ausdifferenzierung des Feldes bei und verändert Evidenzpraktiken folgenreich. Dass sich z.B. für den Nachweis der Sicherheit, zuerst von Kernkraftwerken und später für andere Technikfelder, die Messung von Risiken etablieren konnte, hat auch damit zu tun, dass sich diese Messungen, die umfangreiche Infrastrukturen voraussetzten, zum Geschäftszweig für private Anbieter entwickelten. ${ }^{19}$ So war bei-

17 Olaf Breidbach, Neue Wissensordnungen. Wie aus Informationen und Nachrichten kulturelles Wissen entsteht, Frankfurt am Main 2008.

18 Michael P. Lynch, The Internet of Us. Knowing More and Understanding Less in the Age of Big Data, New York 2016, S. 179.

19 Vgl. dazu Dominic Golding, A Social and Programmatic History of Risk Research, in: Sheldon Krimsky u. Dominic Golding (Hg.), Social Theories of Risk, Westport 1992, S. 23-52. 
spielsweise in den USA neben Norman Rassmussen John Garrick als Mitinhaber einer renommierten US-amerikanischen Firma zur Risikobewertung von Kernkraftwerken ein zentraler Akteur in der Risikoquantifizierung. ${ }^{20}$ Diese Kommodifizierung von Evidenzpraktiken zeigt sich auch in anderen Praxisfeldern, zum Beispiel im Gesundheitswesen. Hier wird die De- und Re-Kontextualisierung von Daten als Rohstoff der Evidenz durch digitale Technologien ermöglicht und durch die Plattformbetreiber realisiert, die an der Vermarktung der Daten interessiert sind. Aber auch Narrative, die ein zentrales Element von Evidenzpraktiken in vielen Bereichen von der Wissenschaft bis in den Journalismus sind, werden nicht selten von kommerziellen Anbietern erstellt. ${ }^{21}$

Ein ganz entscheidender Grund für wachsende Herausforderungen an Evidenzpraktiken liegt schließlich in der kulturellen Pluralisierung der Gesellschaften, aber mehr noch in ihrer wachsenden Spaltung durch Verschärfung der Ungleichverteilung und zunehmend heterogene Lebenswelten. Damit erodiert die Basis für Verständigung. Dass plötzlich alternative Fakten in der Gesellschaft florieren, hat sehr viel mit alternativen (extrem ungleichen) Realitäten, in denen wir leben, zu tun. Darauf hat Latour in seinem jüngsten Buch eindrucksvoll hingewiesen, wo er die Leugnung des Klimawandels mit der zunehmenden sozialen Ungleichverteilung von Einkommen und Lebenschancen in Verbindung bringt. Bei aller Verurteilung der Populisten, die alternative Fakten goutieren, so Latour, darf nicht vergessen werden, dass die Superreichen und obskure Eliten die Menschen mit Modernisierungsversprechen getäuscht haben, von denen sie schon wussten, dass diese nicht mehr für alle zu realisieren sind. „Before accusing the people of no longer believing in anything, one ought to measure the effect of that overwhelming betrayal on people's level of trust. “22 Mit dieser Zerstörung des Vertrauens wird es immer schwerer, auf die Glaubwürdigkeit von Wissen, also Evidenz, zu setzen.

20 Die Firma Pickard, Lowe and Garrick, Inc. hatte bis 198821 probabilistische Risikoanalysen von Kernkraftwerken in den USA durchgeführt. Vgl. dazu B. John Garrick, Lessons Learned from 21 Nuclear Plant Probabilistic Risk Assessments, in: Nuclear Technology 84, H. 3, 1989: https:/static1.squarespace.com/static/5462 8adae4b0f587f5d3e03f/t/54c1dfb9e4b06765d7da5808/1421991865916/Lessons+Le arned + from $+21+$ Nuclear + Plant + Probabilistic + Risk + Assessments.pdf [Stand: 27.8.2019].

21 Lisbeth Clausen, Global News Production, Copenhagen 2003.

22 Bruno Latour, Down to Earth. Politics in the New Climatic Regime, Cambridge, UK 2018, S. $22 \mathrm{f}$. 
Das gilt umso mehr, seit Akteure mit handfesten Wirtschaftsinteressen begannen, die Glaubwürdigkeit von Wissen durch die Pervertierung der Norm des organisierten Skeptizismus zu untergraben. Ein Paradebeispiel dafür sind die Aktivitäten der Tabakkonzerne seit den 1950er Jahren, die Studien in Auftrag gaben, um wissenschaftlich begründete Aussagen über die Schädlichkeit des Rauchens zu konterkarieren. ${ }^{23}$ Ermöglicht wurde das nicht zuletzt infolge eines rasanten Anstiegs der Zahl der Anbieter von Forschungsleistungen, der in vielen Bereichen zu beobachten ist und die Validierung von Ergebnissen schwieriger macht. Diese Flut an industriefreundlichen Studien sollte nicht nur die Öffentlichkeit, sondern auch die Gerichte überzeugen.

Dass Gerichten immer häufiger die Entscheidung strittiger Maßnahmen übertragen wird, macht sie zu wichtigen Akteuren in der Feststellung von Evidenz. Sie haben, z.B. in Auseinandersetzungen um potentiell gefährliche Technik, medizinische Behandlungen oder Evaluierungsentscheidungen usw. zu prüfen, ob die Angeklagten ihre Entscheidungen auf ausreichende Evidenz stützen können. Und sie generieren mit der richterlichen Entscheidung Evidenz für analoge Konfliktfälle und Problemfelder. Wenn sie dabei die Argumente von als Gutachter_innen herangezogenen technischen Expert_innen validieren, fügen sie den Kriterien, nach denen die Plausibilität und Vertrauenswürdigkeit von Argumenten beurteilt wird, Aspekte aus der Praxis der Rechtsprechung hinzu. ${ }^{24}$ Dabei transformieren sie die in anderen Bereichen üblichen Evidenzpraktiken. So hat der für die Beweisführung in Rechtsprozessen konstitutive Skeptizismus einen anderen Charakter als jener innerhalb der Wissenschaft, weil es nicht darum geht, Fehler zu vermeiden, sondern Fehler nachzuweisen. Für die im Gerichtssaal festgestellte Evidenz ist letztendlich die Autorität des Rechtswesens entscheidend, und die Prozesse der Verständigung sind an die hochgradig ritualisierten Abläufe von Gerichtsverfahren gebunden. Das impliziert, dass die Letztentscheidung in Konfliktfällen von fachfremdem (Rechts-)Expert_innen getroffen wird und somit auf einer Form sekundärer, von Gutachter_innen bereitgestellter Evidenz beruht. So wurden in den Auseinandersetzungen um die Sicherheit der Kerntechnik in der Bundesrepublik mehr als in anderen Ländern Entscheidungen an die der Öffentlichkeit nicht rechenschaftspflichtigen Richter_innen delegiert, was

23 Naomi Oreskes u. Erik M. Conway, Merchants of Doubt. How a Handful of Scientists Obscured the Truth on Issues from Tobacco Smoke to Global Warming, London 2010.

24 Ausführlich dazu vgl. Roger Smith u. Brian Wynne, Expert Evidence. Interpreting Science in the Law, London u. New York 1989, S. $23 \mathrm{ff}$. 
die Aushandlungsprozesse um die Evidenz kerntechnischer Sicherheit modifiziert, aber nicht entschärft hat. ${ }^{25}$

Der Schritt in die Öffentlichkeit verändert auch die Darstellung von Evidenz. Wenn Wissenschaftler_innen eigene Forschungsergebnisse ihren Kolleg_innen erklären, werden sie anders argumentieren als in einer öffentlichen Anhörung, wo diese Ergebnisse zur Begründung strittiger Entscheidungen herangezogen werden. Im ersten Falle wird zugelassen, dass Evidenz auch fragil sein kann, während im zweiten Falle eher eindeutige Geltungsbehauptungen aufgestellt werden. Die Zulassung fragiler Evidenz in wissenschaftsinternen Bereichen kann aber auch als Counterevidenz von wissenschaftsexternen Akteur_innen verwendet werden. Das war beispielsweise der Fall, als die E-Mails von Klimaforscher_innen der Universität East Anglia 2009 gehackt und dann veröffentlicht wurden, um die Klimaforschung zu diskreditieren. ${ }^{26}$

\section{Vom Wissen zur Gewissheit: Evidenzpraktiken als Aushandlungsprozesse ${ }^{27}$}

Im Lichte dieser für die Kohäsionskraft gegenwärtiger Gesellschaften nicht ungefährlichen Entwicklungen wird es wichtig zu verstehen, welche Praktiken der Verständigung auf Evidenz demokratische Gesellschaften hervorbringen, um über eine Grundlage für Entscheidungen zu verfügen, und welche Rückwirkungen das sowohl auf die Erzeugung des Wissens selbst als auch auf den gesellschaftlichen Zusammenhalt hat. Im Zentrum dieses Buches steht deshalb die Frage, wie Evidenz gemacht und gebraucht wird. Evidenz wird dabei als sozial konsentiertes Wissen bestimmt, das in Aushandlungsprozessen entsteht. In der Literatur wird dafür auch der Begriff der diskursiven Evidenz verwendet, z.B. von Mittelstraß, der von „der methodisch (durch Beweis, Erklärung etc.) fortschreitenden Einsicht“ spricht. ${ }^{28}$ In diesen Aushandlungsprozessen sind freilich die Forscher_in-

25 James E. Dooley et al., The Management of Nuclear Risk in Five Countries. Political Cultures and Institutional Settings, in: Roger E. Kasperson u. Jeanne X. Kasperson (Hg.), Nuclear Risk Analysis in Comparative Perspective, Boston 1987, S. 27-48, hier S. 39.

26 Richard Holliman, Advocacy in the Tail. Exploring the Implications of 'Climategate' for Science Journalism and Public Debate in the Digital Age, in: Journalism 12, H. 7, 2011, S. 832-846.

27 Teile des folgenden Abschnitts sind in Zusammenarbeit mit Mariacarla Gadebusch Bondio entstanden.

28 Jürgen Mittelstraß, ,Evidenz', in: ders. (Hg.), Enzyklopädie Philosophie und Wissenschaftstheorie, Band 1, Stuttgart 2005, S. 609f., hier S. 609. 
nen nur eine der beteiligten Gruppen. Weitere Akteur_innen und Institutionen kommen hinzu, seit mit der Entfaltung der Wissensgesellschaft mannigfaltige epistemische Gemeinschaften entstehen und seit in den Massendemokratien der Anspruch auf Mitwirkung an der Wissensproduktion und -verwendung geltend gemacht wird.

In diesen Aushandlungsprozessen formieren sich sozioepistemische Arrangements, in denen ganz unterschiedliche Entitäten (Akteure, Diskurse und Materialitäten) zusammenwirken, um den Ergebnissen wissenschaftlicher Verfahren in ihren mannigfaltigen Darstellungsformen durch $\mathrm{Zu}$ stimmung bzw. Verwendung Sinn und damit Glaubwürdigkeit zu attestieren. Mit dem heuristischen Konzept der sozioepistemischen Arrangements erfassen wir mehr als die epistemischen Gemeinschaften, die freilich auch Teil dieser Arrangements sind. Unsere Aufmerksamkeit richtet sich jedoch auch auf die materiellen und virtuellen Artefakte technisierter Wissensproduktion, -darstellung und -vermittlung, die z.B. in der Citizen Science, der Personalisierten Medizin, in den Bereichen der technischen und der Lebensmittelsicherheit, aber auch in der Anthropozändebatte und in der Wissensvermittlung durch die Medien einen aktiven Anteil an der Validierung des Wissens durch unterschiedliche Communities haben.

Evidenzpraktiken implizieren Kommunikationsprozesse, die Bestandteil der Herstellung, Vermittlung und Nutzung von Wissen in der Gesellschaft sind. Sie sind an der sensiblen Schnittstelle angesiedelt, an der die unterschiedlichen und dennoch untrennbar miteinander verknüpften Logiken der Erzeugung und Verwendung von Wissen zusammenkommen. Ebenso wie in der industriellen Warenwirtschaft das Aufeinandertreffen von Produktion und Konsumtion als ein neuralgischer Punkt gilt, den Marx mit der Metapher vom Salto mortale der Waren herausgehoben hat, ist auch in der Wissensgesellschaft der Übergang von der Erzeugung zur Verwendung der Evidenz ein durchaus kritischer Moment. Mit dem Salto mortale vom Wissen zur Gewissheit entscheidet sich, ob neues Wissen verwendet und verwertet werden kann, in der Fortsetzung der Forschung, für den Transfer in neue Wissenschaftsdisziplinen oder in praktische Anwendungsfelder, in der Vermittlung zu Bildungszwecken oder der Begründung von politischen, medizinischen, ökonomischen oder anderen Entscheidungen kollektiver oder individueller Natur. Hier entsteht jene zirkuläre Dynamik, die wir mit der rhetorischen Figur des Chiasmus von practicing evidence und evidencing practice erfassen. Die Validierung von Ergebnissen epistemischer Verfahren erfolgt immer mit Bezug auf deren Nutzungsweise. Gleichzeitig verändern diese Validierungsprozesse aber auch die Herstellung von und die Ansprüche an Evidenz, wenn diese in immer mehr Handlungs- und Entscheidungsfeldern zur legitimatorischen Grundlage 
wird. Evidenz nicht als Repräsentation, sondern als Performanz und Enactment sowohl in der Herstellung wie in der Anwendung zu betrachten, eröffnet uns dabei direkte Zugänge zu den Transformationsprozessen in der spätmodernen Wissensgesellschaft und erlaubt uns, Veränderungen in den Zugängen zu und der Bedeutung von Wissen in den unterschiedlichen Herstellungs-, Vermittlungs- und Verwendungszusammenhängen besser zu verstehen.

Ein wichtiges Anliegen, Evidenz nicht als Repräsentation, sondern als Arrangement von Praktiken zu untersuchen, ist es, die menschlichen und nichtmenschlichen Akteur_innen ins Bild zu rücken, die diese Praktiken aufführen. Dabei wird sichtbar, dass immer mehr und zunehmend auch sehr verschiedene Teilnehmer_innen involviert sind. Ihr spezifisches Vorgehen beim Erklären, Verstehen, Begründen, Rechtfertigen und Akzeptieren oder Ablehnen von Argumenten und Entscheidungen ist in Abhängigkeit vom konkreten Kontext unterschiedlich und für die menschlichen Akteure immer auch interessengebunden. Eine Folge ist die zunehmende Diversität der sozioepistemischen Arrangements und der Evidenzpraktiken, was die der Rede vom sogenannten postfaktischen Zeitalter zugrundeliegende Beobachtung erklären kann, dass das für deliberative Demokratien essentielle Bemühen um Verständigung und Übereinstimmung immer schwerer aufrechtzuerhalten ist.

\section{Wissens- und Machtordnungen: Evidenzpraktiken im Zusammenspiel}

In den Beiträgen dieses Bandes werden Evidenzpraktiken als konkrete Handlungsweisen untersucht und vorgestellt, die in je spezifischen Praxisfeldern in Bezug aufeinander oder in Abgrenzung voneinander aufgeführt werden. Betrachtet werden Ein- und Ausschließen, De- und Re-Kontextualisieren, Messen und Ermessen, Bewerten und Gewichten sowie Erzählen und Analysieren. Die paarweise Verknüpfung, mit der sowohl Unterschiede als auch Komplementaritäten herausgestellt werden, konturiert die einzelnen Praktiken schärfer. Sie macht gleichzeitig gegenseitige Abhängigkeiten, aber auch zeit- und kontextspezifische Priorisierungen deutlich, wie die Fallstudien der Projekte zeigen.

Der Band beginnt mit Institutionen und Akteur_innen sowie der Frage, wer überhaupt zu akzeptierten Wissensbeständen beitragen kann. Das Kapitel Einschließen und Ausschließen verweist auf eine spannungsreiche Verknüpfung, in der die eine Praktik nicht ohne die andere gedacht werden kann. Diese Dynamik der Einbeziehung neuer Akteur_innen, Wissensbestände und Wissensformen bei gleichzeitiger Abgrenzung findet sich zum 
Beispiel in der Citizen Science. Hier erfolgen Einschließungsprozesse als transdisziplinäre Öffnungen, ${ }^{29}$ um durch Partizipationsmöglichkeiten von Nicht-Expert_innen an der Generierung von Wissen dessen soziale und kulturelle Anschlussfähigkeit zu erhöhen und so gesellschaftliche Evidenz im Sinne einer Zustimmung der Öffentlichkeit zu erzeugen. Auch die Anthropozändebatte, das zweite Fallbeispiel des ersten Kapitels, öffnet den Blick auf Spannungsfelder, die aus innerwissenschaftlichen Einschließungs- und Ausschließungsprozessen resultieren. Besonders deutlich werden diese in den Auseinandersetzungen der Anthropocene Working Group über die wissenschaftliche Evidenz für die These vom Anthropozän als neuem geologischen Zeitabschnitt. Dass bei der Organisation solcher trans- und interdisziplinären Einschlüsse aber immer auch Ausschlüsse über Ressourcen, Formen der Arbeitsteilung, Maßnahmen zur Qualitätssicherung oder problematische Simplifizierungen vorgenommen werden, relativiert sowohl den Innovationsgehalt als auch das Ausmaß der erzeugten sozialen Evidenz.

Die Praktiken des De- und Re-Kontextualisierens verweisen auf grundlegende Prozesse wissenschaftlichen Arbeitens selbst. Dieses zweite Kapitel analysiert, wie in Forschungsprozessen Daten erhoben werden, indem man sie aus ihrem Kontext löst. Relevanz erhalten Daten jedoch nicht kontextlos, vielmehr müssen Forschungsergebnisse stets in einem Anwendungszusammenhang gedacht werden, um als wissenschaftlicher Befund wirksam zu werden. Daten fungieren in diesem Sinne als Rohstoff von Evidenz. Nur im Kontext interpretiert werden sie zu wertvollen Informationen, und erst diese „In-Formierungen“ verleihen ihnen Relevanz. ${ }^{30}$ Die Dynamik der Extraktion von Daten und ihrer anschließenden Rekontextualisierung untersucht das Kapitel am Beispiel von Patientenplattformen in der Medizin und von Citizen-Science-Projekten in der Lokalgeschichtsschreibung. Indem sie mit diesen Beispielen Forderungen nach „realitätsnaher Evidenz" nachgehen, geht es also um die Rekontextualisierung von Daten durch Betroffene und Laien. Diese Rekontextualisierung, so die These des Kapitels, ist aber nur dann wirksam, wenn sie auch von der Wissenschaft selbst wahr- und aufgenommen wird.

Im dritten Kapitel Messen und Ermessen geht es um Evidenzpraktiken, die sich auf den ersten Blick durch unterschiedliche Modi des Autorisierens unterscheiden. Messen operiert mit Zahlen und Metriken, die als Me-

29 Transdisziplinärität wird hier im wissenssoziologischen Sinne als eine Kooperation mit Personen außerhalb des Wissenschaftssystems verstanden.

30 Breidbach (2008), S. 108. 
dium der Kommunikation auf Grund ihrer großen Anschlussfähigkeit an das epistemische Normengerüst moderner Wissenschaft (Universalität, Transparenz etc.) besonders dort eine hohe Glaubwürdigkeit genießen, wo das Vertrauen in Zahlen ein Misstrauen in Eliten kompensieren kann. Ermessen hingegen basiert auf Erfahrungswissen und ist an das Vertrauen in Experten gebunden, das in Ungleichheit tolerierenden Kulturen eher kulturell anschlussfähig ist als in Kulturen, die sich auf Offenheit, Transparenz und Zugänglichkeit berufen. Anhand zweier Fallstudien, die sich zum einen mit der Risikoquantifizierung als Evidenz für (kern-)technische Sicherheit und zum anderen mit der Evidenz für Exzellenz bei der Leistungsbewertung von Nachwuchswissenschaftler_innen beschäftigen, wird gezeigt, dass der Einsatz beider Evidenzpraktiken nicht allein sachliche, sondern maßgeblich auch sozio-kulturelle Gründe hat. Die Gutachter_innen von Spitzenwissenschaftler_innen rekurrierten auf das geschulte Expert_innenurteil, um die Expert_innen für die Zukunft zu reproduzieren. Im Unterschied dazu setzten die Protagonist_innen der Risikoquantifizierung auf die hohe Glaubwürdigkeit von Zahlen, um die Gegner_innen der Kerntechnik durch „objektive“ Verfahren zu überzeugen. Dabei sind Messen und Ermessen aber keineswegs einander ausschließende Praktiken, sondern eng miteinander verbunden. Dem Messen liegen immer Ermessenspraktiken zugrunde, die sich auf das Erfahrungswissen und damit die Autorität von Experten stützen. Dass das jedoch verkannt und erst in Evidenzkrisen thematisiert wird, verweist auf die Wirkungsmacht kontextspezifischer Evidenzkulturen, die unterschiedliche Modi des Autorisierens favorisieren. ${ }^{31}$

Das Kapitel Bewerten und Gewichten beschreibt, wie Evidenz als Entscheidungshilfe der Politik nutzbar gemacht wird. Mit Fallbeispielen aus der internationalen Umwelt- und Gesundheitspolitik zeigt es, dass Bewertungen und Gewichtungen vor allem dann relevant sind, wenn Evidenz widersprüchlich ist. So fordert die Komplexität der untersuchten Fälle, beispielsweise der Einsatz von Pestiziden, eine stetige Aushandlung, wie Risikofaktoren und Folgeabschätzungen in die Entscheidungsfindung einbezogen werden sollen. Entscheidungen können eine Vielzahl von Konsequenzen aufweisen, die in ihren einzelnen Dimensionen zu bewerten sind. Die Praktik des Gewichtens rückt dagegen stärker die Folgeabschätzung von Entscheidungen in einem komplexen System durch eine Quantifizie-

31 Vgl. Sheila Jasanoff, Product, Process, or Programme. Three Cultures and the Regulation of Biotechnology, in: Martin Bauer (Hg.), Resistance to New Technology, Cambridge 1995, S. 311-331. 
rung der zugeordneten Relevanzen in den Blick. Das Kapitel erörtert, wie insbesondere die Praktik der Kosten-Nutzen-Analyse zentrales Element für Regulierungen und Interventionen wurde, und diskutiert die Kritik an dieser Entwicklung. Neben dem Ausschluss der Öffentlichkeit, die mit der Verwissenschaftlichung politischer Entscheidungen einhergeht, richtet sich die Kritik vor allem gegen den Ausschluss alternativer Arten der Bewertung sowie gegen intransparente Gewichtung spezifischer Evidenz. Evidenzbasierte und damit auf Kosten-Nutzen-Analysen beruhende Politik löst deshalb das Versprechen nach rationalen und transparenten Entscheidungsstrukturen nur bedingt ein.

Die Praktiken des Erzählens und Analysierens wiederum verwenden grundlegend verschiedene Mittel zur Durchsetzung von Geltungsansprüchen. Narrative benutzen das Medium der Sprache als Kommunikationsform und die jahrtausendealte Kulturtechnik des Geschichten-Erzählens, um Ergebnisse epistemischer Verfahren zu kontextualisieren und damit kulturell anschlussfähig zu machen. Diese Vorgehensweise hat eine große Wirkungsmacht im Journalismus erlangt, der in modernen Gesellschaften zur wichtigsten Quelle der Evidenz für den größten Teil der Bevölkerung geworden ist. ${ }^{32}$ Gleichwohl sind Narrative auch in vielen Wissenschaften grundständige Evidenzpraktiken. ${ }^{33}$ Die Historiker_innen Veronika Lipphardt und Kiran Patel haben das beispielsweise für die Evolutionsbiologie untersucht und „Neuverzauberung“ genannt, womit sie das Verfahren der Narrativierung mit Max Webers Diagnose von der „Entzauberung der Welt" durch die analytisch vorgehende Wissenschaft kontrastieren. ${ }^{34} \mathrm{Im}$ Unterschied zur Narration, die Daten, Fakten, Tatsachen, Erkenntnisse etc. kontextualisiert, um so Einsichten zu generieren, stellt das Verfahren der Analyse durch Dekontextualisierung Geltungsbehauptungen auf. Das analytische Vorgehen galt in der klassischen, erklärenden Wissenschaft als wichtigster Weg zur Erkenntnis und das verschaffte der Analyse ein hohes Prestige als Evidenzpraktik. Dieses Prestige aber hat sich im 20. Jahrhun-

32 „Was wir über unsere Gesellschaft, ja über die Welt, in der wir leben, wissen, wissen wir durch die Massenmedien." Niklas Luhmann, Die Realität der Massenmedien, Opladen 1996, S. 9.

33 Mary S. Morgan u. M. Norton Wise, Narrative Science and Narrative Knowing. Introduction to Special Issue on Narrative Science, in: Studies in History and Philosophy of Science Part A, 62, 2017, S. 1-5; David Gugerli et al. (Hg.), Erzählen, in: Nach Feierabend. Zürcher Jahrbuch für Wissensgeschichte 10, 2014.

34 Veronika Lipphardt u. Kiran Patel, Neuverzauberung im Gestus der Wissenschaftlichkeit. Wissenspraktiken im 20. Jahrhundert am Beispiel menschlicher Diversität, in: Geschichte und Gesellschaft 34, H. 4, 2008, S. 425-454. 
dert im Zusammenhang mit der zunehmenden Thematisierung von Nichtwissen, Risiko und Ungewissheit verringert. Inzwischen bedienen sich Vertreter_innen analytisch vorgehender Wissenschaften auch narrativer Verfahren, um ihren Ergebnissen Plausibilität und Glaubwürdigkeit zu verleihen.

Die paarweise Analyse der Praktiken zeigt zum einen, dass Aushandlungen um Evidenz in einem Geflecht konkurrierender, paralleler oder auch aufeinander aufbauender Handlungen stattfinden. Während beispielsweise Einschließen und Ausschließen gegenläufige Dynamiken zeigen, stellen Bewerten und Gewichten unterschiedliche Stufen von Entscheidungsprozessen ins Zentrum. In beiden Fällen sind aber die Praktiken untrennbar miteinander verknüpft. Evidenzpraktiken sind also stets im Zusammenspiel und nicht als Einzelhandlungen zu betrachten. Oftmals können bestimmte Praktiken insbesondere im Vergleich mit oder als Ergänzung zu anderen überzeugen: Messen und Ermessen werden beispielsweise als Gegensätze gedacht, deutet doch Ermessen auf einen Spielraum hin, den es mit Expertise zu nutzen gilt, während Messen eine streng formalisierte Tätigkeit beschreibt. Dabei ergänzen sich beide Praktiken produktiv: Expert_innen beziehen sich einerseits auf objektive Messergebnisse, interpretieren Sachverhalte aber andererseits auch auf der Grundlage ihrer Ausbildung und Erfahrung. Auch Erzäblen und Analysieren wirken insbesondere in Kontrast zueinander, wobei die Erzählung mit massenmedialer Kommunikation assoziiert wird und die Analyse mit der Wissenschaft. Unsere Untersuchung des Zusammenspiels der Praktiken zeigt jedoch, dass diese Gegensätzlichkeit in der Praxis so nicht unbedingt auszumachen ist. Die Praktik des Messens ist keineswegs so objektiv, sondern wird vielmehr in erheblichem Maße von Auswahl- und Ermessensentscheidungen geprägt. Entsprechend finden sich auch in der scheinbar analytischen Wissenschaft viele narrative Elemente.

Zum anderen verweist die Gesamtschau der Praktiken auf drei zentrale Gemeinsamkeiten, die alle unsere untersuchten Aushandlungsprozesse um die Glaubwürdigkeit, Überzeugungskraft und Gültigkeit von Wissen verbinden.

Erstens geht es in allen Beispielen um das Verhältnis von Wissenschaft, Expert_innen und Öffentlichkeit bzw. verschiedener Teilöffentlichkeiten. Bereits der Mediziner und Wissenschaftstheoretiker Ludwik Fleck unterschied in den 1930er Jahren in seiner Analyse von Denkkollektiven nicht starr zwischen Wissenschaft und Öffentlichkeit, sondern beschrieb einen esoterischen Kreis der Fachspezialisten und einen exoterischen Kreis der interessierten Laien, zwischen denen es eine Reihe von Abstufungen gebe. Dabei wirkt jedoch nicht nur der esoterische Kreis auf die Ränder, sondern 
auch die durch Laien geprägte öffentliche Meinung wirkt auf die Experten zurück. ${ }^{35}$ Auch in der medialisierten Wissensgesellschaft der Gegenwart werden wissenschaftliche Befunde in verschiedenen Teilöffentlichkeiten nicht nur rezipiert, sondern, wie beispielsweise die Anthropozändebatte oder auch die Berichterstattung über Genforschung zeigen, auch transformiert. Was in der Wissenschaft noch als fragliches Konzept verhandelt wird, erhält durch die öffentliche Thematisierung eine Verfestigung und kulturelle Aufladung, der sich in der Folge auch die Wissenschaftler_innen nicht entziehen können. ${ }^{36}$

Wissenschaft und Öffentlichkeiten nutzen sich in den untersuchten Fallbeispielen in vielfältiger Hinsicht „als Ressourcen füreinander“. ${ }^{37}$ Diese Entwicklung deckt sich mit Flecks Befund zum Austausch zwischen Wissenschaft und Laien in demokratischen Gesellschaften: „Die jeweiligen esoterischen Kreise treten hiermit zu ihren exoterischen Kreisen in Beziehung, die wir aus der Soziologie als die der Elite zur Masse kennen. Hat die Masse stärkere Positionen, dann prägt sich dieser Beziehung ein demokratischer Zug auf." 38 So lässt sich in Patientenbewegungen, die aus der Kritik an Evidenzbasierter Medizin entstanden, nicht nur der Einfluss von Laiengruppen auf die Forschung feststellen, sondern auch ihre Legimitationskraft und nicht zuletzt das Interesse an ihren Daten. Die in Bewerten und Gewichten beschriebene Roll-Back-Malaria-Kampagne betreibt erheblichen Aufwand für ihre mediale Außenwirkung und ist in ihren finanziellen Ressourcen auch davon abhängig. Auch die in Erzäblen und Analysieren untersuchten Narrativierungen im Wissenschaftsjournalismus wie in der Wissenschaft zeigen die Tendenz, wissenschaftliche Befunde öffentlichkeitstauglich zu machen. Beide Kapitel verdeutlichen zudem, wie komplex und ausdifferenziert das Beziehungsgeflecht zwischen verschiedenen Wissenschafts- und medialen Teilöffentlichkeiten im 20. Jahrhundert gewor-

35 Ludwik Fleck, Entstehung und Entwicklung einer wissenschaftlichen Tatsache, Frankfurt am Main 1993, S. 148-150.

36 Rudolf Stichweh, The Multiple Publics of Science. Inclusion and Popularization, in: Soziale Systeme 9, H. 2, 2003, S. 210-220; Mike Michael, Publics Performing Publics. Of PiGs, PiPs and Politics, in: Public Understanding of Science 18, H. 5, 2009, S. 617-631. Zur Frage der Transformation von Fakten durch ihre Zirkulation vgl. außerdem Mary S. Morgan, Travelling Facts, in: dies. u. William Peter Howlett (Hg.), How Well Do Facts Travel? The Dissemination of Reliable Knowledge, Cambridge 2011, S. 3-40.

37 Sybilla Nikolow u. Arne Schirrmacher (Hg.), Wissenschaft und Öffentlichkeit als Ressourcen füreinander. Studien zur Wissenschaftsgeschichte im 20. Jahrhundert, Frankfurt am Main 2007.

38 Fleck (1993), S. 139. 
den ist. So sind schließlich mit Wissenschaftsjournalismus, Politikberatung oder UN-Initiativen neue Mittler zwischen beiden Sphären entstanden.

Zweitens ist Evidenz oder die Überzeugungskraft von Wissen in all diesen Feldern fraglich oder ein besonderes Desiderat geworden. Im Fall der Citizen Science oder der Aushandlungen um Evidenzbasierte Medizin findet eine Infragestellung traditioneller Autoritäten statt. Im Fall der Risikoanalyse, der evidenzbasierten Politik oder der europäischen Forschungsförderung wird mit der Entstehung neuer Problemlagen, Märkte und Institutionen ein Desiderat an Evidenz offensichtlich. Eben dieses Bedürfnis nach Evidenz und (neuer) Überzeugungskraft verleiht den untersuchten Praktiken eine gewisse Dringlichkeit und Prekarität. Dabei geht es in allen untersuchten Fällen um weit mehr als die Glaubwürdigkeit der alten oder neuen Experten. Schließlich sind an ihre Deutungshoheit weitreichende politische und finanzielle Entscheidungen geknüpft. Wie die untersuchten Beispiele aus der Wissenschaftslandschaft, der internationalen Energie-, Umwelt- oder Gesundheitspolitik oder der Pharmaindustrie zeigen, geht es bei der Aushandlung um die Gültigkeit von Wissen auch immer um die Verteilung von Ressourcen. Wenn wir also mit Foucault davon sprechen, dass Evidenzpraktiken von Machtkämpfen nicht zu entkoppeln sind, ist damit sowohl der Bezug auf diskursive Macht als auch auf materielle Verteilungskämpfe gemeint.

Als ein Gegentrend zur Destabilisierung von Evidenz und zur Ausdifferenzierung und Pluralisierung der sozioepistemischen Arrangements lässt sich drittens in unseren Fallstudien die Institutionalisierung von Evidenzpraktiken beobachten. Damit werden bestimmte Verfahren, Schritte und Prozeduren festgelegt, die Evidenzfeststellungen transparent und nachvollziehbar machen und gegen Kontroversen immunisieren sollen. Ein Beispiel dafür stellt der Beitrag zum Bewerten und Gewichten vor, zwei Praktiken, die zentral für Kosten-Nutzen-Analysen sind. Deren Geschichte begann in den USA, wo sie entwickelt wurden, um die Entscheidung für öffentliche Projekte des Wasserbaus zu rationalisieren. ${ }^{39} \mathrm{Im}$ Kontext eines hier aus Traditionen des Liberalismus erklärbaren Misstrauens gegenüber dem Staat war die Standardisierung der Verfahren von Kosten-NutzenAnalysen ein probates Mittel, um Entscheidungen auf die Glaubwürdigkeit von Zahlen und das Befolgen von Regeln zu stützen. Einen Spezialfall von Kosten-Nutzen-Analysen stellen Risikoanalysen dar. Kosten-NutzenAnalysen finden seit den 1980er und 90er Jahren in immer mehr Berei-

39 Vgl. dazu Porter (1996), S. 148-189. 
chen weltweit Verwendung, obwohl sie in ihrer standardisierten Form den Bedingungen in konkreten Anwendungsbereichen und -orten, zum Beispiel im Globalen Süden, nur selten gerecht werden können. Sie sind durch ihr konkretes Framing sehr selektiv und schließen Betrachtungen aus, die sich nicht mit den vorgegebenen Verfahren durchführen lassen. Dabei stellen sich auch moralische Fragen zur Angemessenheit der Verfahren, wenn beispielsweise der Wert menschlichen Lebens über Ausfallzeiten in der Produktion ermittelt oder Schadenswirkung als ein Anstieg der Mortalitätsrate quantifiziert wird.

Die Gründung der Anthropocene Working Group im Jahre 2009 stellt eine andere Form der Institutionalisierung von Evidenzierungsverfahren dar. Der provokativen und umwälzenden Wirkung der Anthropozänthematik auf Prozesse innerwissenschaftlicher Wissensproduktion wurde mit dieser Gruppe eine Struktur geschaffen. Sie erfolgt hier über die Regelung von Ein- und Ausschlüssen sowie über die Festlegung konkreter Beweis- und Periodisierungsverfahren. So löste die eigentlich geowissenschaftlich gedachte These eines neuen Zeitalters nicht nur im geistes- und sozialwissenschaftlichen sowie im kulturellen Bereich Interesse aus, sondern auch etablierte Geologen erprobten außerhalb der eigenen Disziplin zu verortende Perspektiven und bezogen neue Kategorien und fachfremde Argumentationslinien in ihre Überlegungen ein. Mit der Gründung einer Working Group wurden also nicht nur ein Konzept, sondern auch neue Evidenzpraktiken institutionalisiert.

\section{Fazit: De- und Re-Stabilisierung von Evidenz}

Was aber bedeuten unsere Befunde für die Verfahren zur Gewinnung von Wissen und seiner sozialen Konsentierung? Unsere Analyse unterschiedlichster Evidenzpraktiken ergibt dazu ein auf den ersten Blick widersprüchliches Bild. So lässt sich die beschriebene Destabilisierung, Transformation und Diversifikation der Wissenslandschaft epistemisch keineswegs ausmachen. Vielmehr bleiben die Verfahren und Methoden zur Herstellung von Evidenz ausgesprochen stabil. Unsere Fallstudien zeigen, dass neue Akteure wie Citizen Scientists, Politikberater oder internationale Organisationen eher etablierte Wissens- und Kommunikationswege kopieren und re-enacten, anstatt neue Standards zu setzen. Wenn wir mit dem Gesellschaftstheoretiker Niklas Luhmann zwischen Sozial-, Sach- und Zeitdimensionen unterscheiden, wird also deutlich, dass sich hier auf verschiede- 
nen Ebenen gegenläufige Tendenzen vollziehen. ${ }^{40}$ Während in der Sozialdimension eine Transformation stattfindet und neue Akteur_innen zugelassen, Autoritäten hinterfragt und alte Strukturen aufgebrochen werden, lässt sich in der Sachdimension vergleichsweise wenig Bewegung feststellen: Die Praktiken der Wissenschaftler_innen und Expert_innen, die Verfahren, mit denen Wissen den Status von Evidenz erlangt, haben sich schließlich eher verfestigt denn transformiert. Diese Stabilisierung von Evidenzpraktiken, so das Argument des vorliegenden Bandes, erfolgt jedoch nicht trotz, sondern gerade wegen der Destabilisierung auf der Ebene der Strukturen: Die Öffnung der Sozialdimension vollzieht sich bei gleichzeitiger Schließung der Sachdimension. Hierin zeigt sich erstens der Drang neuer Akteure, ein Feld zu besetzen und akzeptiert zu werden, also sozial konsentiertes Wissen anbieten zu können. Zweitens spiegelt sich in der Reglementierung der Sachdimension der Versuch etablierter Wissenschaftler_innen und Expert_innen, (wenigstens) die epistemische Kontrolle zu behalten bzw. wiederzuerlangen.

\section{Literatur}

Böschen, Stefan, Zur Einleitung. Fragile Evidenz - Wissenspolitischer Sprengstoff, in: Technikfolgenabschätzung - Theorie und Praxis 22, H. 3, November 2013, S. 4-9.

Böschen, Stefan u. Wehling, Peter, Neue Wissensarten. Risiko und Nichtwissen, in: Sabine Maasen et al. (Hg.), Handbuch Wissenschaftssoziologie, Wiesbaden 2012, S. 317-327.

Breidbach, Olaf, Neue Wissensordnungen. Wie aus Informationen und Nachrichten kulturelles Wissen entsteht, Frankfurt am Main 2008.

Clausen, Lisbeth, Global News Production, Copenhagen 2003.

Dooley, James E. et al., The Management of Nuclear Risk in Five Countries. Political Cultures and Institutional Settings, in: Roger E. Kasperson u. Jeanne X. Kasperson (Hg.), Nuclear Risk Analysis in Comparative Perspective, Boston 1987, S. 27-48.

Fleck, Ludwik, Entstehung und Entwicklung einer wissenschaftlichen Tatsache, Frankfurt am Main 1993.

Funkenstein, Amos, Theology and the Scientific Imagination from the Middle Ages to the Seventeenth Century, Princeton 1986.

40 Niklas Luhmann, Soziale Systeme. Grundriß einer allgemeinen Theorie, Frankfurt am Main 1984, S. 111-122. 
Garrick, B. John, Lessons Learned from 21 Nuclear Plant Probabilistic Risk Assessments, in: Nuclear Technology 84, H. 3, 1989: https://static1.squarespace.com/st atic/54628adae4b0f587f5d3e03f/t/54c1dfb9e4b06765d7da5808/1421991865916/L essons + Learned + from $+21+$ Nuclear + Plant + Probabilistic + Risk + Assessments.pdf [Stand: 27.8.2019].

Golding, Dominic, A Social and Programmatic History of Risk Research, in: Sheldon Krimsky u. Dominic Golding (Hg.), Social Theories of Risk, Westport 1992, S. 23-52.

Gugerli, David et al. (Hg.), Erzählen, in: Nach Feierabend. Zürcher Jahrbuch für Wissensgeschichte 10, 2014.

Holliman, Richard, Advocacy in the Tail. Exploring the Implications of 'Climategate' for Science Journalism and Public Debate in the Digital Age, in: Journalism 12, H. 7, 2011, S. 832-846.

Jasanoff, Sheila, Product, Process, or Programme. Three Cultures and the Regulation of Biotechnology, in: Martin Bauer (Hg.), Resistance to New Technology, Cambridge 1995, S. 311-331.

Kaiser, Mario, Neue Zukünfte - Gegenwarten in Verzug, in: Sabine Maasen et al. (Hg.), Handbuch Wissenschaftssoziologie, Wiesbaden 2012, S. 395-408.

Latour, Bruno, Down to Earth. Politics in the New Climatic Regime, Cambridge, UK 2018.

Latour, Bruno, Die Hoffnung der Pandora, Frankfurt am Main 2000.

Lepore, Jill, After the Fact. In the History of Truth, a New Chapter Begins, in: The New Yorker, 14.3.2016: https:/www.newyorker.com/magazine/2016/03/21/the-i nternet-of-us-and-the-end-of-facts [Stand: 24.4.2019].

Lipphardt, Veronika u. Patel, Kiran, Neuverzauberung im Gestus der Wissenschaftlichkeit. Wissenspraktiken im 20. Jahrhundert am Beispiel menschlicher Diversität, in: Geschichte und Gesellschaft 34, H. 4, 2008, S. 425-454.

Luhmann, Niklas, Die Realität der Massenmedien, 2. Auflage, Opladen 1996.

Luhmann, Niklas, Soziale Systeme. Grundriß einer allgemeinen Theorie, Frankfurt am Main 1984.

Lynch, Michael P., In Praise of Reason, Cambridge u. London 2012.

Lynch, Michael P., The Internet of Us. Knowing More and Understanding Less in the Age of Big Data, New York 2016.

Michael, Mike, Publics Performing Publics. Of PiGs, PiPs and Politics, in: Public Understanding of Science 18, H. 5, 2009, S. 617-631.

Mittelstraß, Jürgen, ,Evidenz', in: ders. (Hg.), Enzyklopädie Philosophie und Wissenschaftstheorie, Band 1, Stuttgart 2005, S. 609-610.

Morgan, Mary S., Travelling Facts, in: dies. u. William Peter Howlett (Hg.), How Well Do Facts Travel? The Dissemination of Reliable Knowledge, Cambridge 2011, S. 3-40.

Morgan, Mary S. u. Wise, M. Norton, Narrative Science and Narrative Knowing. Introduction to Special Issue on Narrative Science, in: Studies in History and Philosophy of Science Part A, 62, 2017, S. 1-5. 
Nikolow, Sybilla u. Schirrmacher, Arne (Hg.), Wissenschaft und Öffentlichkeit als Ressourcen füreinander. Studien zur Wissenschaftsgeschichte im 20. Jahrhundert, Frankfurt am Main 2007.

Oreskes, Naomi u. Conway, Erik M., Merchants of Doubt. How a Handful of Scientists Obscured the Truth on Issues from Tobacco Smoke to Global Warming, London 2010.

Porter, Theodore, Trust in Numbers. The Pursuit of Objectivity in Science and Public Life, Princeton 1996.

Raspe, Heiner, Eine kurze Geschichte der Evidenz-basierten Medizin in Deutschland, in: Medizinhistorisches Journal 53, H. 1, 2018, S. 71-82.

Rescher, Nicholas, Ignorance. On the Wider Implications of Deficient Knowledge, Pittsburgh 2009.

Schiemann, Gregor, Wahrheitsgewissheitsverlust. Hermann von Helmholtz' Mechanismus im Anbruch der Moderne. Eine Studie zum Übergang von klassischer zu moderner Naturphilosophie, Darmstadt 1997.

Schulz-Schaeffer, Ingo u. Böschen, Stefan, Einleitung, in: Stefan Böschen et al. (Hg.), Wissenschaft in der Wissensgesellschaft, Wiesbaden 2003, S. 9-23.

Smith, Roger u. Wynne, Brian, Expert Evidence. Interpreting Science in the Law, London u. New York 1989.

Stichweh, Rudolf, The Multiple Publics of Science. Inclusion and Popularization, in: Soziale Systeme 9, H. 2, 2003, S. 210-220.

Weiland, Sabine, Evidenzbasierte Politik zwischen Eindeutigkeit und Reflexivität, in: Technikfolgenabschätzung - Theorie und Praxis 22, H. 3, November 2013, S. 9-15.

Weingart, Peter, „Wahres Wissen“ und demokratisch verfasste Gesellschaften, in: Aus Politik und Zeitgeschichte (APuZ) 67, H. 13, 2017, S. 11-16.

Wilde, Jessica, Ulrich Beck: Die Risikogesellschaft als Wegbereiter der Wissensgesellschaft?, in: Anina Engelhardt u. Laura Kajetzke (Hg.), Handbuch Wissensgesellschaft. Theorien, Themen und Probleme, Bielefeld 2010, S. 35-42. 
